\title{
Prevalence of Impaired Glucose Tolerance and Diabetes Mellitus in Women with Polycystic Ovarian Syndrome
}

\author{
${ }^{1} \mathrm{CH}$ Chandra Smitha, ${ }^{2}$ Mo Orkoth Prasannan Anantha Sailakshmi, ${ }^{3}$ Rangaiah Nagaratnamma
}

\begin{abstract}
In women of reproductive age polycystic ovary syndrome (PCOS) is the common endocrine disorder. It is one of the common causes of infertility due to anovulation. There is no single criterion for the diagnosis of this syndrome. It is crucial to diagnose PCOS early in its course since it has lifelong implications with increased risk for metabolic syndrome, type II Diabetes mellitus (DM), cardiovascular disease, endometrial hyperplasia, infertility and depression. Screening for the impaired glucose (IGT), allows for proper and timely prevention and management of complications. In this cross sectional study, we planned to detect the prevalence of impaired glucose tolerance and diabetes in women with PCOS which will facilitate prevention of long term sequelae. We also studied the extent of the risk for diabetes in patients with PCOS in relation to age, socioeconomic status, rural and urban areas, BMI, waist/hip circumference ratio, family history of PCOS and diabetes mellitus
\end{abstract}

Keywords: Diabetes mellitus, Impaired glucose tolerance, Polycystic ovarian syndrome.

How to cite this article: Smitha CHC, Sailakshmi MOPA, Nagaratnamma R. Prevalence of Impaired Glucose Tolerance and Diabetes Mellitus in Women with Polycystic Ovarian Syndrome. J Med Sci 2017;3(4):116-118.

Source of support: Nil

Conflict of interest: None

\section{INTRODUCTION}

Polycystic ovarian syndrome is one of the commonest endocrine metabolic disorders affecting 4 to $12 \%$ of women in their reproductive age. ${ }^{1}$ It is the most common cause of infertility due to anovulation. ${ }^{1}$ The disease exists as a genetic predisposition in the person and its symptoms are exacerbated by environmental factors and lifestyle. ${ }^{2}$ The disease begins soon after puberty mostly at 15 to 25 years of age and manifests during the reproductive period. ${ }^{3}$ It is primarily characterized by ovulatory dysfunction and hyperandrogenism. The risk for diabetes has been seen to be higher in women with PCOS. Age of

\footnotetext{
${ }^{1}$ Postgraduate Student, ${ }^{2}$ Professor, ${ }^{3}$ Professor and Head

${ }^{1-3}$ Department of Obstetrics and Gynecology, RajaRajeswari Medical College \& Hospital, Bengaluru, Karnataka, India
}

Corresponding Author: $\mathrm{CH}$ Chandra Smitha, Postgraduate Student, Department of Obstetrics and Gynecology RajaRajeswari Medical College \& Hospital, Bengaluru Karnataka, India, e-mail: chandra.smithareddy@gmail.com onset of diabetes is significantly earlier in these women, who are more likely to experience gestational diabetes. ${ }^{4}$ It is crucial to diagnose PCOS early in its course since it has lifelong implications with increased risk for metabolic syndrome, type II DM, cardiovascular disease, endometrial hyperplasia, infertility, and depression. ${ }^{5}$ Screening for the IGT allows for proper and timely prevention and management of complications. ${ }^{6}$ Multiple risk factors contribute to diabetes obesity, centripetal fat distribution, insulin resistance, hyperinsulinemia, decreased peripheral insulin sensitivity, beta-cell dysfunction, chronic anovulation, dyslipidemia, and family history of type DM II. ${ }^{1}$ About 7.5 to $10 \%$ have type II diabetes and 30 to $35 \%$ have IGT. ${ }^{1}$ The IGT is a significant risk factor for developing diabetes. ${ }^{7}$ The rate of conversion from IGT to type II DM will be increased by 5 to 15 -fold in PCOS. ${ }^{7}$

In this cross-sectional study, we planned to detect the prevalence of IGT and diabetes in women with PCOS so as to facilitate prevention of long-term sequelae. We also studied the extent of the risk for diabetes in patients with PCOS in relation to age, socioeconomic status, rural and urban areas, body mass index (BMI), waist/hip circumference ratio, family history of PCOS, and DM. After the Institutional Ethical Committee's approval, we recruited 100 women diagnosed to have PCOS according to Rotterdam criteria 2003.

The PCOS was diagnosed when at least two of the below-mentioned criteria were present:

- Oligo- or anovulation (oligomenorrhea, amenorrhea)

- Clinicaland/orbiochemical signs of hyperandrogenism

- Polycystic ovaries on ultrasound (at least one ovary with increased ovarian volume $>10 \mathrm{~cm}^{3}(\mathrm{~mL})$ or $\geq 12$ follicles measuring $2-9 \mathrm{~mm}$ in diameter)

We excluded other conditions that mimic PCOS.

We also excluded patients with DM, hypothyroidism, hyperprolactinemia, Cushing syndrome, nonclassical adrenal hyperplasia, virilizing adrenal tumors or ovarian tumors, current or previous use of drugs like oral contraceptives, synthetic progestogens having androgenic activity, androgens, glucocorticoids, antidiabetic drugs, sodium valproate, and phenytoin.

\section{MATERIALS AND METHODS}

The present study includes 100 subjects diagnosed as PCOS among women presenting to the Department of 
Obstetrics and Gynaecology, RajaRajeshwari Medical College \& Hospital, India.

\section{Methods}

Informed consent was obtained from all the subjects after explaining the nature and purpose of the study. All the patients were subjected to detailed history and clinical examination using predesigned proforma. Data pertaining to their age, place, socioeconomic status, health status, menstrual history, hirsutism, acne, weight gain, height, weight, food habits, lifestyle, family history of PCOS, and DM were obtained. The prevalence was reflected in terms of percentages and proportions.

The following laboratory investigations were carried out:

- Hemoglobin\%, total count, differential count, erythrocyte sedimentation rate

- Oral glucose tolerance test (OGTT)

- Ultrasound pelvis

- Transabdominal scan in unmarried women

- Transvaginal scan in married women.

Uterus, endometrial thickness, volume of both ovaries, evidence of polycystic ovaries, or any pelvic pathology was observed for:

- Thyroid profile

- Serum prolactin

- Serum testosterone

- Lipid profile.

\section{Oral Glucose Tolerance Test}

- All the subjects are advised to take normal diet for 3 days and overnight fasting for 10 hours.

- Fasting plasma glucose levels and plasma glucose level 2 hours after oral administering 75 grams of glucose in $200 \mathrm{~mL}$ of water will be measured by glucose oxidase method.

- Glucose levels were interpreted according to World Health Organization (WHO) criteria (Table 1).

The WHO glycemic criteria for diagnosis of different categories of glucose intolerance by 75 gm, 2 hours OGTT.

\section{Statistical Analysis}

Data were analyzed using the Statistical Package for the Social Sciences software (version 19.0, SPSS Statistics). Frequencies and percentages were used for categorical data.

Table 1: WHO criteria

\begin{tabular}{lll}
\hline Criteria & $F P G, m g / d L$ & $\begin{array}{l}2-h r \text { postglucose, } \\
\mathrm{mg} / \mathrm{dL}\end{array}$ \\
\hline Normal glucose tolerance & $<110$ & $<140$ \\
Impaired fasting glucose & $100-125$ & - \\
Impaired glucose tolerance & - & $140-199$ \\
Diabetes mellitus & $\geq 126$ and/or & $\geq 200$ \\
\hline
\end{tabular}

\section{RESULTS}

Out of 100 women, 73 women had normal glucose metabolism (NGM) and 27 women had altered glucose metabolism. Of this, 22 women had impaired glucose metabolism and 5 had DM (Table 2). Out of 100 patients, 15 were 15 to 25 years of age and 85 were 25 to 35 years of age.

\section{SOCIOECONOMIC STATUS}

Rural: 23 (31.50\%) belonged to NGM, 7 (31.81\%) were in IGT, and $0(0.00 \%)$ in DM (Table 3).

Urban: 50 (68.40\%) belonged to NGM, $15(68.18 \%)$ were in IGT, and 5 (100\%) in DM (Table 3).

Incidence of oligomenorrhea, hirsutism, acne, infertility, and acanthosis nigricans has been shown in Table 4.

Incidence of BMI in NGM, IGT, and DM subjects is depicted in Table 5.

Waist/hip ratio circumference in NGM and DM subjects is shown in Table 6. Totally, 17 in the NGM, 14 in IGT, and 4 in the DM group had $\mathrm{W} / \mathrm{H}$ ratio $>0.80$.

Table 2: Age group

\begin{tabular}{llll}
\hline Age (in years) & NGM (\%) & IGT $(\%)$ & $D M(\%)$ \\
\hline $15-25$ & $13(18.00)$ & $2(9.09)$ & $0(0.00)$ \\
$25-35$ & $60(82.00)$ & $20(90.09)$ & $5(100.00)$ \\
\hline
\end{tabular}

Table 3: Socioeconomic status

\begin{tabular}{llll}
\hline Grade & NGM (\%) & IGT (\%) & $D M(\%)$ \\
\hline I & $11(15.06)$ & $3(13.61)$ & $1(20.00)$ \\
II & $23(31.50)$ & $2(0.09)$ & $2(40.00)$ \\
III & $22(30.13)$ & $9(40.9)$ & $1(20.00)$ \\
IV & $17(23.28)$ & $8(36.36)$ & $1(20.00)$ \\
\hline
\end{tabular}

Table 4: Features of PCOS

\begin{tabular}{llll}
\hline Features & NGM (\%) & IGT (\%) & DM (\%) \\
\hline Oligomenorrhea & $64(87.67)$ & $20(90.9)$ & $5(100.00)$ \\
Hirsutism & $38(52.05)$ & $14(63.63)$ & $3(60.00)$ \\
Acne & $31(42.26)$ & $10(45.45)$ & $3(60.00)$ \\
Infertility & $41(56.16)$ & $16(72.72)$ & $4(80.00)$ \\
Acanthosis nigricans & $19(26.02)$ & $12(54.54)$ & $3(60.00)$ \\
\hline
\end{tabular}

Table 5: Incidence of BMI

\begin{tabular}{clll}
\hline BMI $\left(\mathrm{kg} / \mathrm{m}^{2}\right)$ & NGM (\%) & IGT $(\%)$ & $D M(\%)$ \\
\hline$<18.5$ & $2(66.60)$ & $1(4.54)$ & $0(0.00)$ \\
$18.5-24.99$ & $40(95.23)$ & $2(9.09)$ & $0(0.00)$ \\
$25-29.99$ & $19(65.51)$ & $9(40.90)$ & $2(40.00)$ \\
$30-34.99$ & $10(58.82)$ & $6(27.27)$ & $2(40.00)$ \\
$35-39.99$ & $2(22.22)$ & $4(18.18)$ & $1(20.00)$ \\
\hline
\end{tabular}

Table 6: Waist/hip $(\mathrm{W} / \mathrm{H})$ circumference ratio

\begin{tabular}{llll}
\hline W/H ratio & NGM (\%) & IGT $(\%)$ & $D M(\%)$ \\
\hline$\leq 0.80$ & $56(76.00)$ & $8(36.36)$ & $1(20.00)$ \\
$>0.80$ & $17(23.00)$ & $14(63.63)$ & $4(80.00)$ \\
\hline
\end{tabular}


Table 7: Family history of DM and PCOS

\begin{tabular}{llll}
\hline Family history & NGM (\%) & IGT (\%) & DM (\%) \\
\hline DM & $16(21.90)$ & $10(45.45)$ & $3(60.60)$ \\
PCOS & $9(12.32)$ & $4(18.18)$ & $1(20.00)$ \\
\hline
\end{tabular}

Subjects with family history of DM and PCOs are shown in Table 7. DM group: 16 (21.90\%) NGM, 10 (45.45\%) IGT and $3(60.60 \%)$ DM. PCOS group: 9 (12.32\%) NGM, 4 (18.18\%) IGT and 1 (20.00\%) DM.

\section{DISCUSSION}

In our study, out of 100 women, 73 women had NGM and 27 women had altered glucose metabolism. Of this, 22 women had impaired glucose metabolism and 5 had DM (Table 2). Out of 100 patients, 15 were in the 15 to 25 years of age and 85 were in the 25 to 35 years of age.

Type II DM is a global public health problem and its prevalence differs widely among the various populations. ${ }^{4}$ Pathogenesis of the disease involves a combination of impaired insulin secretion, insulin resistance, and genetic and environmental factors. ${ }^{6}$ Type II DM is the end of a long metabolic derangement and IGT is a prediabetic glucose intolerant stage characterized by insulin resistance. ${ }^{5}$

The risk for diabetes has been seen to be higher in women with PCOS. ${ }^{6}$ Thus, women with PCOS have a substantially higher prevalence of IGT and diabetes than in age- and weight-matched healthy women. ${ }^{7}$ The PCOS is associated with insulin resistance and hyperinsulinemia. ${ }^{8}$

Improvement in insulin sensitivity resulted in the improvement of metabolic and gynecological parameters, such as oligomenorrhea and facilitation of pregnancies. ${ }^{9}$ Age of onset of diabetes is significantly earlier in these women and they are more likely to experience gestational diabetes. ${ }^{10}$

A number of therapies have been successfully used for the treatment of insulin resistance in PCOS, including metformin and trioglitazone.,11

The IGT is a relatively asymptomatic stage and patients may survive without any symptoms for a long time. But, in women with PCOS, manifestations of androgen excess, disordered gonadotropin secretion, and postbinding defect in insulin signaling usually bring the patients for clinical examinations and may lead to early detection of IGT. ${ }^{12}$

\section{CONCLUSION}

The PCOS is not only an endocrine reproduction disease, but also is a metabolic disorder. The IGT and type II DM are more common in PCOS patients. The prevalence of IGT and type II DM was higher in women with PCOS than expected in the general population. Since insulin resistance is a key factor in the incidence of type II diabetes, it is suggested that women with PCOS are at a higher risk for developing type II diabetes. Women with PCOS should periodically have an OGTT and must be closely monitored for deterioration in glucose tolerance.

\section{REFERENCES}

1. Aali B, Naderi T. Evaluation of clinical, ultrasound and laboratory features of PCOS in Kerman in 1381. Iran J Endocrinol Metab 2004 Jun;6(2):153-161.

2. Conway GS, Hanorr JW, Jacobs HS. Heterogensity of the polycystic ovary syndrome: clinical, endocrine and ultrasound feature in 556 patients. Clin Endocrinol (Oxf) 1989 Apr;30(4):459-470.

3. Flannery CA, Rackow B, Cong X, Duran E, Selen DJ, Burgert TS. PCOS in adolescence: impaired glucose tolerance occurs across the spectrum of BMI. Pediatr Diabetes 2013 Feb;14(1):42-49.

4. Lankarani M, Valizadeh N, Heshmat R, Shafaee AR, Amini MR, Noori M, Aleyasin A, Larijani B. Evaluation of dyslipidemia in polycystic ovary syndrome. J Diabetes Metab Disord 2005;4(2):E11+E11i-E11+E11x.

5. Pfeifer SM, Kives S. Polycystic ovary syndrome in the adolescent. Obstet Gynecol Clin North Am 2009 Mar;36(1): 129-152.

6. Biyasheva A, Legro RS, Dunaif A, Urbanek M. Evidence for association between polycystic ovary syndrome (PCOS) and TCF7L2 and glucose intolerance in women with PCOS and TCF7L2. J Clin Endocrinol Metab 2009 Jul;94(7):2617-2625.

7. Bhattacharya SM. Abnormal glucose tolerance in polycystic ovary syndrome. J Obstet Gynaecol Res 2008 Apr;34(2):228-232.

8. Mor E, Zograbyan A, Saadat P, Bayrak A, Tourgeman DE, Zhang C, Stanczyk FZ, Paulson RJ. The insulin resistant subphenotype of polycystic ovary syndrome: clinical parameters and pathogenesis. Am J Obstet Gynecol 2004 Jun;190(6):1654-1660.

9. Velazquez EM, Mendoza S, Hamer T, Sosa F, Glueck CJ. Metformin therapy in polycystic ovary syndrome reduces hyperinsulinemia, insulin resistance, hyperandrogenemia and systolic blood pressure while facilitating normal menses and pregnancy. Metabolism 1994 May;43(5):647-654.

10. Mirzaei F, Kazemi N. Investigate the prevalence of PCOS in women with type two DM, visiting Kerman diabetes clinic. Sci J Hamadan Univ Med Sci 2007;14:38-42.

11. Ünlühizarc K, Kele timur F, Bayram F, Sahin Y, Tutu A. The effects of metformin on insulin resistance and ovarian steroidogenesis in women with polycystic ovary syndrome. Clin Endocrinol (Oxf) 1999 Aug;51(2):231-236.

12. Farhadifar F, Lotfi M, Naseri K, Moghimi N, Yaghma'ee $\mathrm{R}$, Rahimi A. Assessing the association between PCOS and glucose intolerance. Sci J Kurdistan Univ Med Sci 2006;11:45-55. 